\title{
Recent Developments of the Modelica "Buildings" Library for Building Energy and Control Systems
}

\author{
Michael Wetter, Wangda Zuo, Thierry Stephane Nouidui \\ Simulation Research Group, Building Technologies Department, \\ Environmental Energy Technologies Division, Lawrence Berkeley National Laboratory, \\ Berkeley, CA 94720, USA
}

\begin{abstract}
At the Modelica 2009 conference, we introduced the Buildings library, a freely available Modelica library for building energy and control systems [16].

This paper reports the updates of the library and presents example applications for a range of heating, ventilation and air conditioning (HVAC) systems. Over the past two years, the library has been further developed. The number of HVAC components models has been doubled and various components have been revised to increase numerical robustness.

The paper starts with an overview of the library architecture and a description of the main packages. To demonstrate the features of the Buildings library, applications that include multizone airflow simulation as well as supervisory and local loop control of a variable air volume (VAV) system are briefly described. The paper closes with a discussion of the current development.
\end{abstract}

Keywords: building energy systems, heating, ventilation, air-conditioning, controls

\section{Introduction}

Buildings account for a large portion of energy consumption and related green house gas emissions. For example, in the United States, buildings consume $2 / 3$ of electricity and $40 \%$ of total energy [4]. In order to reduce global green house gas emissions, it is critical to reduce building energy consumption by increasing energy-efficiency and by using more renewable energy. To support the design and operation of low energy buildings, a simulation program should support:

1. rapid prototyping of new building systems,

2. comparison of the performance of different designs of the building, its energy system and its control algorithms,

3. analysis of the operation of existing building systems,

4. development and specification of building control sequences, and

5. reuse of models during operation for energyminimizing controls, fault detection and diagnostics.

To support these use cases, we develop an open-source Modelica library for building energy and control systems. The library is freely available from http://www.modelica.org/ libraries/Buildings.

At the 7th Modelica conference in 2009, we introduced the Buildings library, version 0.6.0, which had 73 non-partial models and blocks, as well as 26 functions. The latest version, 0.10 .0 , has 129 non-partial models and blocks and 39 functions. This paper highlights some of these updates to inform users about the new capabilities.

The paper is structured as follows: Section 2 gives an overview of the Buildings library. Section 3 describes the updates in detail. Section 4 presents applications with models for multizone airflow simulation and for co-simulation. Section 5 describes classes which are currently under development and will be available in future releases.

\section{Summary of the Buildings Li- brary}

The Buildings library is based on the Modelica.Fluid library [8]. The Buildings library is organized into the packages shown in Fig. 1. Components in these packages augment models from the Modelica Standard Library and from the Modelica.Fluid library. Base classes, which are typically not of interest to the end-user, but are used to construct other classes, are stored in packages called BaseClasses. Most packages 
contain a package called Examples, which contains example applications. The examples illustrate typical use of components in the parent directories. They are also used to conduct unit tests.

The current Buildings library contains six major packages, including a new package Airflow. The package Airflow provides models to compute the airflow inside the buildings and between the building and the ambient environment. It currently has a package Multizone for multizone airflow models, which is discussed in Section 3.1. The Controls package contains components for continuous time control, discrete time control and scheduling of set points. The package Fluid is the largest package of the Buildings library. It contains components for fluid flow systems, such as pumps, boilers, chillers, valves and sensors, which are described in Section 3.3. The package HeatTransfer contains models for heat transfer in buildings (Section 3.4). It provides models and functions for heat transfer due to convection, conduction and radiation. It also has thermal property data for different solid materials. The Media package contains media models that are simpler and generally computationally more efficient than the ones in the Modelica Standard Library. The simplifications have been done by taking into account that HVAC systems in buildings typically have a smaller range of operating conditions compared to other thermodynamic applications. The Utilities package provides utilities such as for the calculation of thermal comfort and psychrometric properties. It also has an interface to the Building Control Virtual Test Bed (BCVTB) [17], which can connect Modelica to other simulation programs, such as EnergyPlus, MATLAB/Simulink and Radiance, for cosimulation. The BCVTB can also connect Modelica models to building automation systems for model-based operation.

\section{Updates of the Buildings Li- brary}

This section compares the newest Buildings library, version 0.10 .0 , with version 0.6 .0 which was reported in the last Modelica conference [16]. The purpose is to explain to users the new packages, models and blocks, as well as their new features.

\subsection{Package Airflow}

The airflow package provides models for computing air flow inside a building and between a building and its exterior environment. For build-

\author{
Buildings.Airflow.Multizone \\ .Controls. Continuous \\ .Discrete \\ SetPoints \\ .Fluid.Actuators.Dampers \\ .Motors \\ .Valves \\ .Boilers \\ .Chillers \\ .Delays \\ .FixedResistances \\ HeatExchangers \\ .HeatExchangers.CoolingTowers \\ .Radiators \\ .Interfaces \\ .MassExchangers \\ .MixingVolumes \\ .Movers \\ .Sensors \\ .Sources \\ .Storage \\ .Utilities \\ .HeatTransfer \\ .Media.ConstantPropertyLiquidWater \\ .GasesPTDecoupled \\ .GasConstantDensity \\ .IdealGases \\ .PerfectGases \\ .Utilities.Comfort \\ .Diagnostics \\ .IO. $B C V T B$ \\ .Math \\ .Psychometrics \\ .Reports
}

Figure 1: Package structure of the Buildings library. Only the major packages are shown. Bold indicates a new package and italic indicates existing packages with new models added.

ing simulation, many different indoor airflow models are generally used, such as multizone network models [3], zonal models [12], computational fluid dynamics [13], and fast fluid dynamics [18]. For indoor airflow simulation, multizone network models with the well-mixed air assumption are fast but not appropriate if the air is stratified. By solving the Navier-Stokes equations and equations for conservation of mass and energy, computational fluid dynamics (CFD) is the most detailed and accurate modeling method. However, computing time for CFD is large for flow simulation in a large building or over a long time horizon. To fill the gap between multizone and CFD, fast fluid dynamics models solve the Navier-Stokes equations with simplified schemes that are much faster but less accurate than CFD. 
Currently, the Airflow package contains multizone airflow models in the package Multizone. These models compute the airflow and contaminant transport between different rooms, as well as between a room and the exterior. Multizone airflow models assume that air and contaminants in each room volume are completely mixed. The driving force for the air flow is pressure difference induced by flow imbalance of the HVAC system, density difference across large openings (such as open doors or windows), stack effects in high rise buildings, and wind pressure on the building facade.

The air volume in each room is modeled by an instantaneously mixed volume that provides differential equations for conservation of mass, species concentration, trace substances and internal energy. As in Modelica.Fluid, a parameter can be used to switch between steady-state and transient simulation, and to switch the initialization equations between steady-state initialization and prescribed state variables.

The flow resistance between these volumes is computed using the orifice equation

$$
\dot{V}=C_{d} A \sqrt{2 / \rho} \Delta P^{m},
$$

where $\dot{V}$ is the volume flow rate, $C_{d}$ is the dimensionless discharge coefficient, $A$ is the cross section area of the opening, $\rho$ is the density of the fluid, $\Delta P$ is the static pressure difference and $m$ is the flow exponent. Large openings are characterized by $m$ very close to 0.5 , while values near 0.65 have been found for small crack-like openings. Typical values for $C_{d}$ and $m$ can be found in [15] and in the citations therein. For pressure differences that are smaller in magnitude than a user-specified parameter, equation (1) is regularized to ensure that it is differentiable with a continuous derivative.

The model EffectiveAirLeakageArea computes air leakage. It describes a one-directional pressure driven air flow through a crack-like opening. The opening is modeled as an orifice. The orifice area is parameterized by processing the effective air leakage area, the discharge coefficient and pressure drop at a reference condition. The effective air leakage area can be obtained, for example, from the ASHRAE fundamentals [1]. A similar model is also used in the multizone airflow modeling software CONTAM [5].

To compute the bi-directional flow across large openings, such as doors, the opening is discretized along its height into compartments. Then, the orifice equation (1) is used to compute the flow for each compartment as explained in [15]. The model DoorDiscretizedOpen describes a door that is always open, and the model DoorDiscretizedOperable describes a door whose opening area can be changed using a control signal.

To model the pressure difference caused by stack effect, one can use the model MediumColumn for a steady-state and MediumColumnDynamic for a transient model. The model MediumColumn computes the pressure difference at its ports using

$$
\Delta p=h \rho g,
$$

where $h$ is the height of the medium column, $\rho$ is the density and $g$ is the earth acceleration. The model MediumColumn can be parameterized to use for $\rho$ the density of either port, or the density of the inflowing medium. The latter situation allows, for example, modeling of a vertical shaft, such as a chimney, whose density may be equal to the one of the inflowing medium. The model MediumColumnDynamic contains, in addition to (2), also a mixing volume that may be used to approximate the transient response of the medium column, or to inject heat into the air stream as may happen in a solar chimney in which walls absorb solar radiation and heat the fluid inside the chimney to increase the buoyancy force.

The models ZonalFlow_ACS and ZonalFlow_m_flow can be used to exchange a fixed flow rate between two volumes. As an input, they use the air exchange rate per second and the mass flow rate, respectively.

The Multizone package was implemented based on the multizone package described in [15], which has been contributed by the United Technologies Research Center (UTRC) for inclusion in the Buildings library. However, several changes have been done when migrating the models to Modelica 3.1, which led to a simpler implementation based on the stream function [9]. A comparison between the two implementations is described in Section 4.1.

\subsection{Package Controls}

The package Controls contains blocks that can be used in conjunction with the controls models from the Modelica Standard Library to implement controllers of building energy systems. The package Controls.Continuous has a new model LimPID, which can provide P, PI, PD, and PID controllers with limited output, anti-windup compensation and setpoint weighting. The package Controls. SetPoints has a new model Table, which allows setting a time-varying set point. 


\subsection{Package Fluid}

The Fluid package contains component models for thermo-fluid flow systems. The level of modeling detail is comparable with the models of the Modelica.Fluid library. Most models in Buildings.Fluid extend models from Modelica.Fluid to form components that are typically needed when modeling building energy systems. The Fluid package is the largest package in the Buildings library, and it has 15 subpackages. This section will discuss seven subpackages to which new models have been added.

\subsubsection{Package Fluid.Chillers}

The package Fluid. Chillers contains two new chiller models. The first chiller model is an electric chiller based on the EnergyPlus chiller model Chiller:Electric:EIR. This model uses three functions to predict its capacity and its power consumption:

- a biquadratic function is used to predict its cooling capacity as a function of condenser entering and evaporator leaving fluid temperature,

- a quadratic function is used to predict its power input to cooling capacity ratio as a function of the part load ratio,

- a biquadratic function is used to predict its power input to cooling capacity ratio as a function of condenser entering and evaporator leaving fluid temperature.

The second implemented chiller model is an electric chiller based on the model by Hydeman et al. [10]. This model is also implemented in EnergyPlus as the model Chiller:Electric:ReformulatedEIR and is similar to the first chiller model. The main difference is that to compute its performance, this model uses the condenser leaving temperature instead of the entering temperature, and it uses a bicubic polynomial instead of a quadratic function to compute the part load performance. This model is reported to provide higher accuracy for variable-speed compressor drive and variable condenser water flow applications compared to the model Chiller:Electric:EIR.

The package Fluid.Chillers.Data contains performance data for more than 300 chillers.

\subsubsection{Package Fluid.Interfaces}

Similarly to Modelica.Fluid.Interfaces, there is a package Buildings.Fluid. Interfaces. It contains partial models for algebraic and dynamic components that exchange heat or mass with one or two fluid streams. The model PartiallumpedVolume has been added to provide a base class for an ideally mixed fluid volume with the ability to store mass and energy. This model is similar to the partial model PartiallumpedVolume from Modelica.Fluid.Interfaces, except that it allows modeling the air humidity using a differential equation, while modeling the total mass balance using a steady-state equation.

\subsubsection{Package Fluid.Actuators}

The package Fluid.Actuators contains models of actuators. There are models of valves with two and three fluid ports and with various opening characteristics as well as models of air dampers. There are also models of motors that can be used in conjunction with the actuators.

The main change to this package was a redesign of the three-way-valves. The new implementation allows the optional addition of a fluid volume where the two fluid streams mix. The fluid volume can be conditionally added or removed based on the parameter dynamicBalance. The use of this fluid volume often leads to a more robust and faster simulation.

\subsubsection{Package Fluid.HeatExchangers}

This package contains algebraic and dynamic heat exchanger models, some of which compute condensation of water vapor that may occur at a cooling coil. Several new models have been added. For example, the model HeatExchangers. DryEffectivenessNTU describes a heat exchanger without water vapor condensation that is based on the effectivenessNTU relation [11]. This model transfers heat in the amount of

$$
\dot{Q}=\epsilon \dot{Q}_{\max }
$$

where $\dot{Q}_{\max }$ is the maximum heat that can be transferred, and $\epsilon$ is the heat transfer effectiveness, defined as

$$
\epsilon=f(N T U, Z, \text { flowRegime }),
$$

where $N T U$ is number of transfer units, $Z$ is the ratio of minimum to maximum capacity flow rate and flowRegime is the heat exchanger flow regime, such as parallel flow, cross flow or counter flow.

Also new in this package are the models DryCoilCounterFlow and WetCoilCounterFlow, which are finite volume models of counter flow heat exchanger without and with water vapor condensation if the air is cooled below its saturation temperature. 


\subsubsection{Package Fluid.Movers}

This package contains component models for fans and pumps. Four new models have been added that can be parameterized by performance curves that compute pressure rise, electrical power draw or efficiency as a function of the flow rate. The four models differ in their implementation of the input signal, which can be a control signal, a prescribed speed, a prescribed mass flow rate or a prescribed pressure rise.

The models FlowMachine $y$ and FlowMachine_Nrpm take a control signal or a number of revolutions as an input, and then compute the resulting pressure difference for the current flow rate. The models FlowMachine_dp and FlowMachine_m_flow take the pressure difference or the mass flow rate as an input signal. The pressure difference or the mass flow rate will then be provided by the fan or the pump. These two models do not have a performance curve for the flow characteristics, because solving for the flow rate and the revolution at zero pressure difference can lead to a singularity.

All models can be configured to have a fluid volume at the low-pressure side. Adding such a volume sometimes helps the solver find a solution during initialization and time integration of large models.

All models compute the motor power draw $P_{\text {ele }}$, the hydraulic power input $W_{\text {hyd }}$, the flow work $W_{f l o}$ and the heat dissipated into the medium $\dot{Q}$. The governing equations are

$$
\begin{aligned}
W_{f l o} & =|\dot{V} \Delta p|, \\
W_{\text {hyd }} & =W_{\text {flo }}+\dot{Q}, \\
\eta & =W_{\text {flo }} / P_{\text {ele }}=\eta_{\text {hyd }} \eta_{\text {mot }}, \\
\eta_{\text {hyd }} & =W_{\text {flo }} / W_{\text {hyd }}, \\
\eta_{\text {mot }} & =W_{\text {hyd }} / P_{\text {ele }},
\end{aligned}
$$

where $\dot{V}$ is the volume flow rate, $\Delta p$ is the pressure rise, $\eta$ is the overall efficiency, $\eta_{h y d}$ is the hydraulic efficiency, and $\eta_{m o t}$ is the motor efficiency. All models take as a parameter an efficiency curve for the motor. This function has the form $\eta_{\text {mot }}=f\left(\dot{V} / \dot{V}_{\max }\right)$, where $\dot{V}_{\max }$ is the maximum flow rate. The models FlowMachine_y and FlowMachine_Nrpm set $\dot{V}_{\max }=f_{c}\left(\Delta p=0, r_{N}=\right.$ 1) where $f_{c}(\cdot, \cdot)$ is a user-specified flow characteristic and $r_{N}$ is the ratio of actual to nominal speed. Since FlowMachine_dp and FlowMachine_m_flow are not parametrized by the function $f_{c}(\cdot, \cdot)$, the parameter $\dot{V}_{\max }$ must be set by the user for these models.
These models have similar parameters as the models in the package Modelica.Fluid.Machines. However, the models in this package differ primarily in the following points:

- They use a different base class, which allows having zero mass flow rate for the fan and pump models. The models in Modelica.Fluid. Machines restrict the number of revolutions, and hence the flow rate, to be non-zero.

- For the model with prescribed pressure, the input signal is the pressure difference between two ports, and not the absolute pressure at the outlet port.

- The pressure calculations are based on total pressure in Pascals instead of the pump head in meters. This change has been made to avoid ambiguities in the parameterization if the models are used as a fan with air as the medium. The original formulation in Modelica.Fluid.Machines converts head to pressure using the density of the medium. For fans, head would be converted to pressure using the density of air. However, manufacturers of fans typically publish the head in millimeters water (mmH20). Therefore, to avoid confusion when using these models with media other than water, our implementation uses total pressure in Pascals instead of head in meters.

- Additional performance curves have been added to the package Buildings.Fluid.Movers. BaseClasses.Characteristics.

\subsubsection{Package Fluid.Sensors}

This package consists of idealized sensor components that provide variables of the medium. These signals can be further processed with components of the Modelica.Blocks library. One can also build more realistic sensor models by further processing their output signal (e.g., by attaching the block Modelica.Blocks.FirstOrder to model the time constant of the sensor). The new version of the library increased the number of sensors from 4 to 22. Sensors are now available for density, enthalpy, mass flow rate, volume flow rate, pressure, relative humidity, dry bulb temperature, wet bulb temperature, species concentration, such as water vapor, and trace substances, such as carbon dioxide. 


\subsubsection{Package Fluid.Sources}

This package contains models for fixed or prescribed boundary conditions for thermo-fluid systems. The new version of the library adds five different combinations of boundary sources. For example, the model Boundary_ph prescribes pressure, specific enthalpy, mass fraction and trace substances. The model MassFlowSource_T is an ideal flow source that produces a prescribed mass flow rate with prescribed temperature, mass fraction and trace substances.

\subsubsection{Package Fluid.Storage}

This package contains models of thermal energy storage tanks. For the model StratifiedEnhanced, a new model using the QUICK scheme [6] for the discretization of the fluid volume has been implemented. It computes a heat flux that needs to be added to each volume in order to give the results that a third-order upwind discretization scheme would give. If a standard third-order upwind discretization scheme were to be used, then the temperatures of the elements that face the tank inlet and outlet ports would overshoot by a few tenths of a Kelvin. To reduce this overshoot, the model uses a first order scheme at the boundary elements, and it adds a term that ensures that the energy balance is satisfied. Without this term, small numerical errors in the energy balance, introduced by the third order discretization scheme, would occur.

\subsection{Package HeatTransfer}

This package contains models for heat transfer elements. Based on a single-layer conduction model ConductorSingleLayer, the new version of the library adds the model ConductorMultiLayer for one-dimensional dynamic and steady-state heat conduction through multi-layer constructions. In addition, a convection model Convection and a model for combination of convection and conduction for opaque constructions ConstructionOpaque are also implemented. The models for heat convection are parameterized by different functions that compute heat convection based on temperature differences, or based on a constant value. These functions are available in the package HeatTransfer.Functions. ConvectiveHeatFlux.

\subsection{Package Media}

This package contains media models that can be used in addition to the models from Modelica.Media. Some of the media models in this package are based on simplified state equations and property equations. The simplification can generally lead to a faster and more robust simulation compared to the models of Modelica.Media. The new version adds a sub-package Media.GasesConstantDensity. The models in this sub-package use a constant mass density to avoid having pressure as a state variable in mixing volumes. The advantage of using constant mass density is that fast transients introduced by a change in pressure are avoided. The disadvantage is that the dimensionality of the coupled nonlinear equation system is typically larger for flow networks.

\subsection{Package Utilities}

This package contains various utility models and functions, including diagnostics models, mathematical functions and a package for cosimulation with the Building Controls Virtual Test Bed (BCVTB). The BCVTB is a middle-ware that can connect Modelica with different simulation programs, such as MATLAB/Simulink, EnergyPlus and Radiance. The BCVTB can also link to building automation systems through a BACnet interface [2] and through analog/digital converters.

More interfaces, such as a model for the boundary condition for HVAC systems that use a medium with moist air BCVTB.MoistAirInterface and blocks for temperature conversions BCVTB.to_degC, BCVTB.from_degC, are introduced in the new version to facilitate the communication between Modelica and the BCVTB. For illustration, Fig. 2 shows an air-based heating system with an ideal heater and an ideal humidifier in the supply duct. The heating system is coupled to the BCVTB for co-simulation with EnergyPlus. The heater and humidifier are controlled with a feedback loop that tracks the room air temperature and room air humidity. These quantities are simulated in the EnergyPlus building simulation program. The component bouBCVTB models the boundary between the domain that models the air system (simulated in Modelica) and the room response (simulated in EnergyPlus).

To assess comfort conditions in rooms, the new version of the library adds a sub-package Comfort. This package contains a model that computes the thermal comfort according to the relations of Fanger [1]. In Fanger's model, the thermal sensation of a human is mainly related to the thermal 


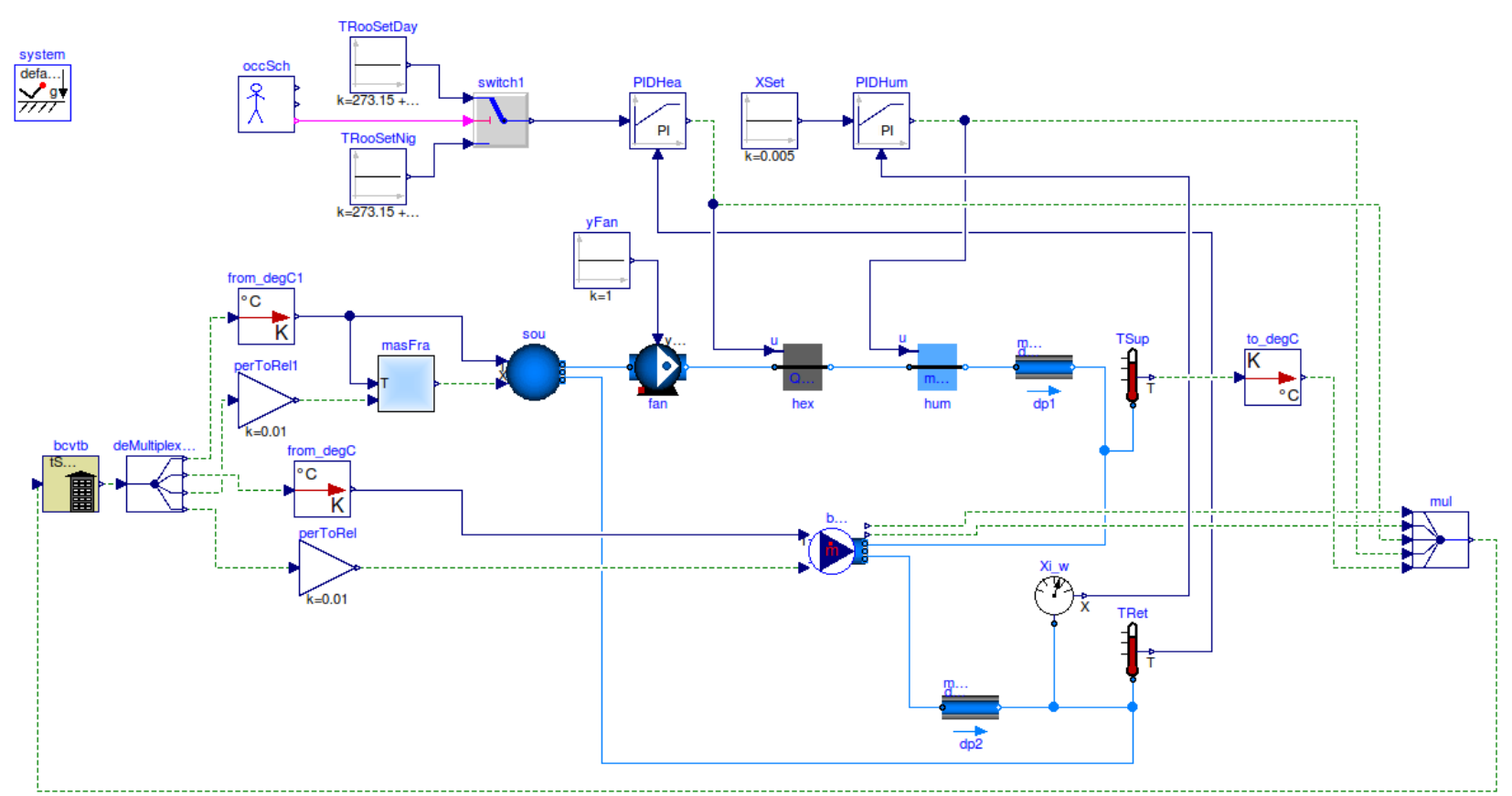

Figure 2: Modelica model that is used for co-simulation of a simple HVAC system that is connected to an EnergyPlus building model.

balance of its body. This balance is influenced by two groups of factors: personal and physical. The activity level and clothing thermal insulation of the subject form the group of personal factors, while the environmental parameters (e.g., air temperature, mean radiant temperature, air velocity, and air humidity) compose the group of physical factors. When the personal factors have been estimated and the physical factors have been measured, the average thermal sensation of a large group of people can be predicted by calculating the PMV (Predicted Mean Vote) index. The PPD (Predicted Percentage of Dissatisfied) index, obtained from the PMV index, provides information on thermal discomfort (thermal dissatisfaction) by predicting the percentage of people likely to feel too hot or too cold in the given thermal environment.

\section{Applications}

\subsection{Multizone Air Flow Model}

The multizone air flow model in the Buildings library is based on the library implemented by UTRC, which is presented in [15]. We converted the library to use the stream connectors [9], and implemented it in the Buildings library. Fig. 3 compares model diagrams for modeling airflow in three rooms. The diagram using stream connectors in Fig.3(a) is much simpler than the one with- out the stream connectors in Fig. 3(b). The models became simpler, since with stream connectors, the inStream operator can be used to obtain the properties of the medium inside the volumes that are connected to the model that computes the stack effect.

We also compared the mass flow rates through the door and the openings in the walls. The mass flow rates computed by the new version are the same as in the original implementation [15], which indicates that the implementation of the multizone airflow models using stream connectors was successful.

\subsection{Modelica EnergyPlus Co- simulation for the Control of a VAV System}

This example illustrates the use of co-simulation between Modelica and EnergyPlus through the BCVTB. In Modelica, we implemented a variable air volume flow system for a building with five thermal zones. The Modelica model implements the airflow network, the fans and heat exchangers, and the supervisory and local loop control. At the air inlet and outlet of the five thermal zones, an energy balance is made for the sensible and latent heat exchange. These heat flows are then added to the model of the thermal zones in EnergyPlus. EnergyPlus then computes the new room temperatures and water vapor concentrations, as 


\section{sylem}

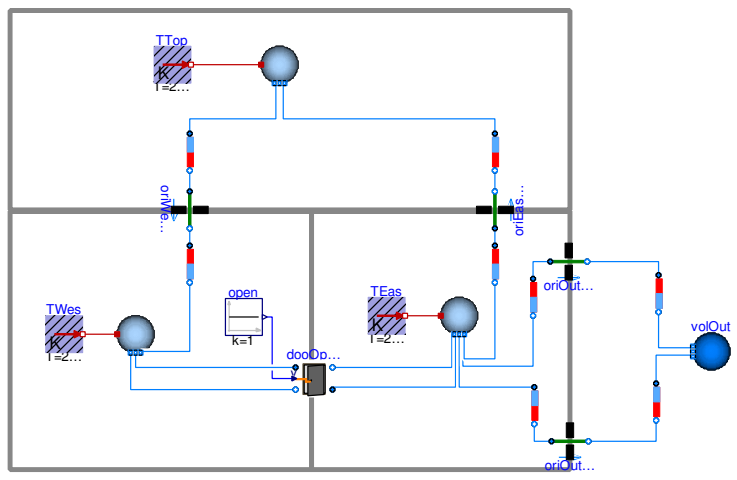

(a) With stream connectors.

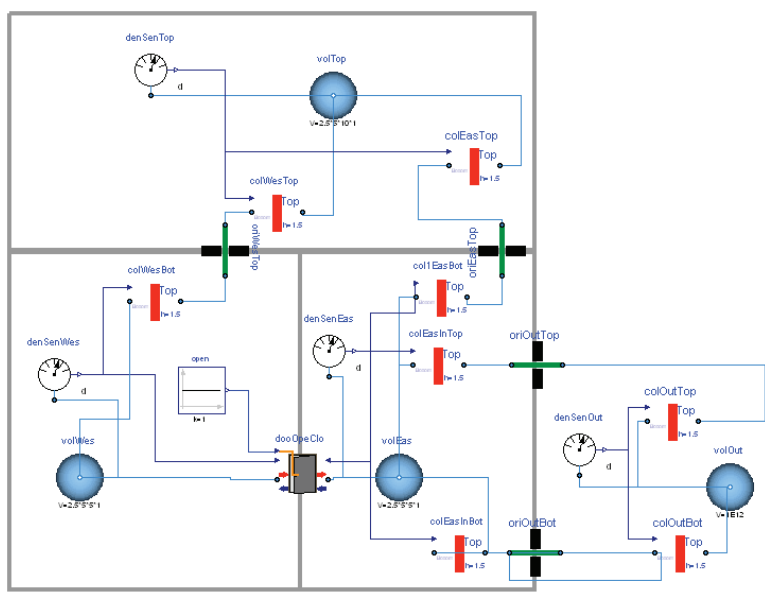

(b) Without stream connectors.

Figure 3: Comparison between diagrams of three room problem implemented by Modelica multizone airflow models with and without stream connectors. Subfigure (b) is the model presented in $[15]$.

well as the current weather conditions. EnergyPlus also computes the heat balance of the building and the lighting control as a function of the available daylight. Thus, the co-simulation allows users to utilize Modelica for the implementation and performance assessment of control sequences, and to utilize EnergyPlus for its whole building heat transfer and daylighting models.

Fig. 4 illustrates the HVAC system as implemented in Modelica. The total system model contained 970 components that led to a differential algebraic equation system with 5,000 scalar equations. The translated model had 90 continuous time states and 20 nonlinear systems of equations with dimensions up to 6 . There are no numer- ical Jacobians. The Modelica models are linked through the BCVTB to EnergyPlus, as shown in Fig. 5. For a more detailed description about this system and its simulation results, see [17].

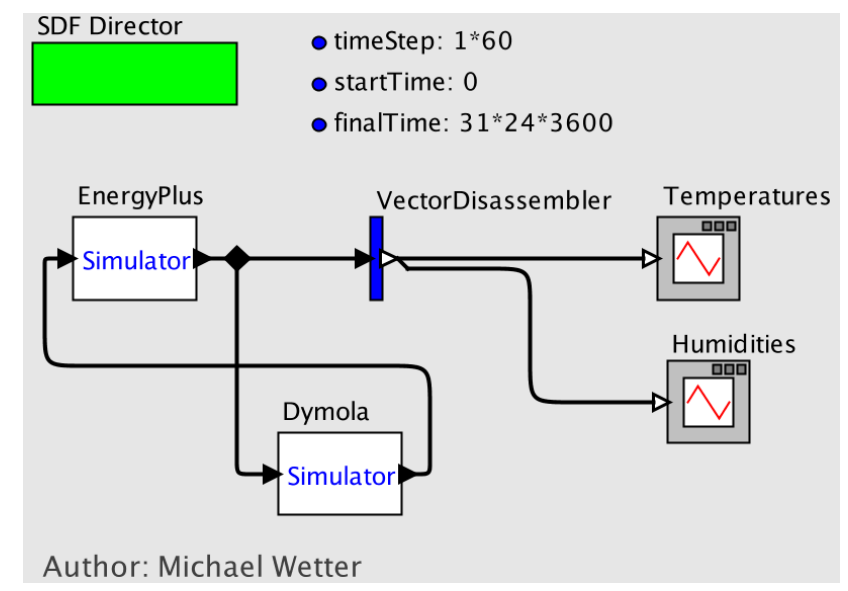

Figure 5: BCVTB model for the co-simulation between Modelica and EnergyPlus.

\section{Ongoing Work}

The next major addition to the Buildings library will be a package with models for a thermal zone that compute heat transfer through the building envelope and within a room. While the Buildings library can already be linked to EnergyPlus, for some situations, it is more practical to have all models implemented in Modelica. This requires the implementation of such a room model that can be used to assemble buildings with several thermal zones.

For the building envelope, we have implemented models for heat conduction through opaque multilayer materials, which are available in the package Buildings. HeatTransfer. Currently, we are working on the implementation of models for window systems. The window model will compute a layer-by-layer radiation, convection and conduction heat balance, using equations that are similar to the ones in the Window 5 program [7].

We are also working on the implementation of models for short-wave, long-wave and convective heat transfer in a room. These models will be similar to the models described in [14].

$$
\text { To obtain boundary conditions, }
$$
we have implemented a package Buildings.BoundaryConditions, which will be released in the next version. This package includes models for the sky black-body temperature, the path of the sun, and incidence angles on tilted surfaces. There will also be a weather 


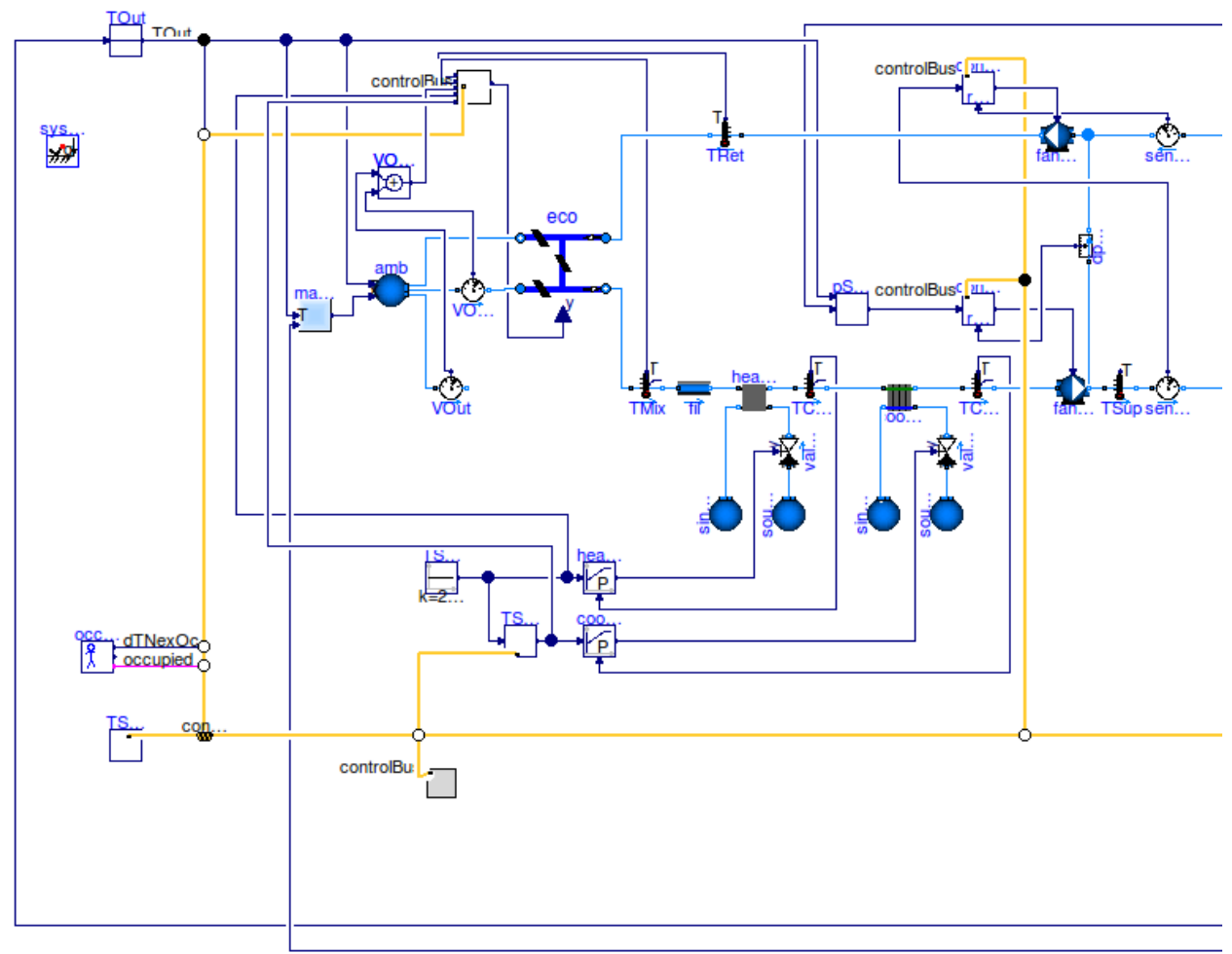

(a) Plant of VAV system.

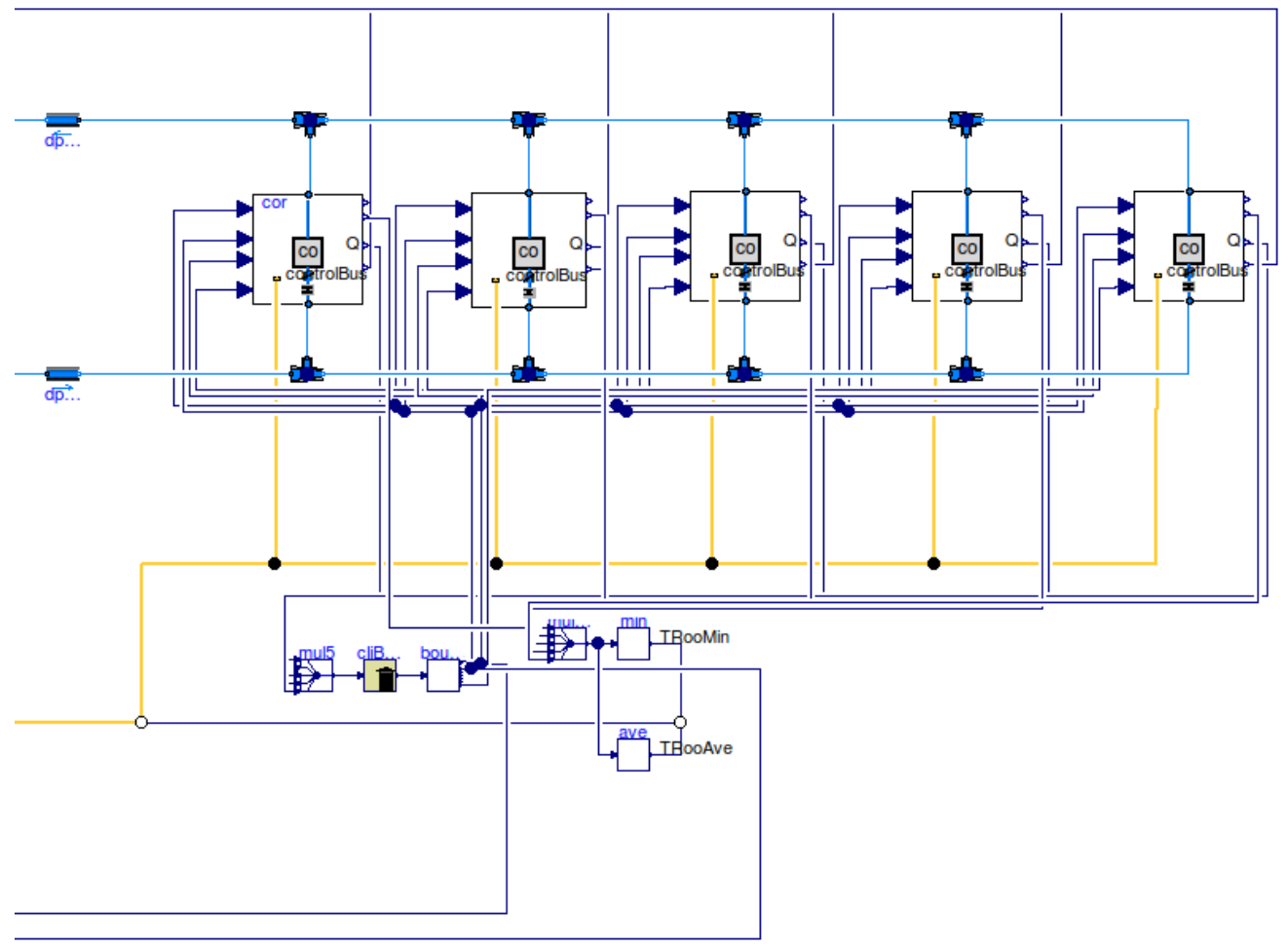

(b) Distribution of VAV system with five thermal zones.

Figure 4: Modelica model of the VAV system that is linked to EnergyPlus for co-simulation. 
data reader. Weather data can be obtained for free from http://www.energyplus.gov, and then converted to Modelica format with a Java program that is provided with the Buildings library.

\section{Conclusion}

The Modelica Buildings library has been significantly expanded since the last Modelica conference. A new Airflow package has been added for indoor air flow simulation. Many models have been added into existing packages to provide more functionality, and existing models have been revised to improve the numerical efficiency. However, for large fluid flow systems with feedback control, such as the ones shown in Fig. 4, computing consistent initial conditions and performing the time integration can still lead to numerical problems. The robust simulation of such systems still requires further research.

\section{Acknowledgments}

This research was supported by the Assistant Secretary for Energy Efficiency and Renewable Energy, Office of Building Technologies of the U.S. Department of Energy, under Contract No. DEAC02-05CH11231.

We would also like to thank the United Technologies Research Center for contributing the Multizone package to the Buildings library.

\section{References}

[1] ASHRAE. ASHRAE fundamentals, 1997.

[2] ASHRAE. ANSI/ASHRAE Standard 135-2004, BACnet, a data communication protocol for building automation and control networks, 2004.

[3] J. Axley. Multizone airflow modeling in buildings: History and theory. HVACER Research, 13(6):907-928, 2007.

[4] DOE. Buildings energy data book. Technical Report DOE/EE-0325, Department of Energy, 2009.

[5] W. S. Dols and G. N. Walton. CONTAMW 2.0 user manual, multizone airflow and contaminant transport analysis software. Technical Report NISTIR 6921, National Institute of Standards and Technology, 2002.

[6] J. H. Ferziger and M. Peric. Computational methods for fluid dynamics. Springer, Berlin, New York, 3rd, rev. edition, 2002.

[7] E. U. Finlayson, D. K. Arasteh, C. Huizenga, M. D. Rubin, and M. S. Reilly. WINDOW
4.0: Documentation of Calculation Procedures. Lawrence Berkeley National Laboratory, Berkeley, CA, USA, 1993.

[8] R. Franke, F. Casella, M. Otter, K. Proelss, M. Sielemann, and M. Wetter. Standardization of thermo-fluid modeling in modelica.fluid. In F. Casella, editor, Proc. of the 7-th International Modelica Conference, Como, Italy, Sept. 2009.

[9] R. Franke, F. Casella, M. Otter, M. Sielemann, H. Elmqvist, S. E. Mattsson, and H. Olsson. Stream connectors: an extension of modelica for device-oriented modeling of convective transport phenomena. In F. Casella, editor, the rth International Modelica Conference, Como, Italy, 2009.

[10] M. Hydeman, N. Webb, P. Sreedharan, and S. Blanc. Development and testing of a reformulated regression-based electric chiller model. ASHRAE Transactions, 108(2), 2002.

[11] F. P. Incropera and D. P. D. Witt. Fundamentals of Heat and Mass Transfer. John Wiley \& Sons, 5th edition, 2001.

[12] A. C. Megri and F. Haghighat. Zonal modeling for simulating indoor environment of buildings: Review, recent developments, and applications. HVACERR Research, 13(6):887-905, 2007.

[13] P. V. Nielsen. Computational fluid dynamics and room air movement. Indoor Air, 14:134-143, 2004.

[14] M. Wetter. Simulation-Based Building Energy Optimization. PhD thesis, University of California, Berkeley, 2004.

[15] M. Wetter. Multizone airflow model in modelica. In the 5th International Modelica Conference, pages 431-440, Vienna, Austria, 2006.

[16] M. Wetter. Modelica library for building heating, ventilation and air-conditioning systems. In the rth International Modelica Conference, Como, Italy, 2009.

[17] M. Wetter. Co-simulation of building energy and control systems with the building controls virtual test bed. In press: Journal of Building Performance Simulation, 2010.

[18] W. Zuo and Q. Chen. Real-time or faster-thanreal-time simulation of airflow in buildings. Indoor Air, 19(1):33-44, 2009. 